\title{
Analytical Void Fraction Profile Near the Walls in Low Reynolds Number Bubbly Flows in Pipes: Experimental Comparison and Estimate of the Dispersion Coefficient
}

\author{
Olivier Marfaing ${ }^{1 *}$, Mathieu Guingo ${ }^{2}$, Jérôme-Marcel Laviéville ${ }^{2}$ and Stéphane Mimouni ${ }^{2}$ \\ ${ }^{1}$ Den-Service de Thermo-Hydraulique et de Mécanique des Fluides (STMF), CEA, Université Paris-Saclay, 91191 Gif-sur-Yvette - France \\ 2 Electricité de France R\&D Division, 6 Quai Watier, 78400 Chatou - France \\ e-mail: olivier.marfaing@cea.fr \\ * Corresponding author
}

\begin{abstract}
In a recent paper, we derived an analytical expression for the void fraction profile in low Reynolds number bubbly pipe flows, based on a balance of hydrodynamic forces on bubbles. The objective of the present work is to perform a comparison of this analytical Bubble Force Balance Formula (BFBF) with an experiment from the literature. We begin by simulating this experiment with the NEPTUNE_CFD code. In particular we show that using an $\mathrm{R}_{\mathrm{ij}}-\varepsilon$ model to account for the liquid velocity fluctuations yields reasonable results. In order to compare our analytical profile with experimental measurements, we restrict ourselves to the near-wall region. In this region, the void fraction profile results from a balance between dispersion and wall forces, and the dispersion coefficient can be considered as uniform. The analytical BFBF profile is seen to be in good agreement with the measurements. We are also capable to estimate the dispersion coefficient in this near-wall region.
\end{abstract}

Résumé - Profil analytique de taux de vide en proche paroi dans les écoulements en conduite à bulles à faible nombre de Reynolds : comparaison expérimentale et estimation du coefficient de dispersion - Dans une publication récente, nous avons obtenu une expression analytique pour le profil de taux de vide dans un écoulement en conduite à bulles à bas nombre de Reynolds, basée sur un bilan des forces hydrodynamiques sur les bulles. L'objectif du travail est d'effectuer une comparaison de cette Formule de Bilan des Forces sur les Bulles (FBFB) avec une expérience de la littérature. Nous commençons par simuler cette expérience avec le code NEPTUNE_CFD. En particulier, nous montrons qu'en utilisant un modèle $R_{i j}-\varepsilon$, nous obtenons des résultats acceptables pour les fluctuations de vitesse liquide. Ensuite, afin de comparer notre profil analytique avec les mesures expérimentales, nous nous restreignons à la zone de proche paroi. Dans cette région, le profil de taux de vide résulte de l'équilibre entre forces de dispersion et de paroi, et le coefficient de dispersion peut être considéré comme uniforme. Le profil analytique FBFB est en bon accord avec les mesures et nous sommes capables d'estimer le coefficient de dispersion dans la zone de proche paroi. 


\section{NOTATIONS}

BFBF Bubble Force Balance Formula

$B \quad$ Constant in Equation (16), determined from the knowledge of the average void fraction over a cross-section

$C_{\mathrm{D}} \quad$ Drag coefficient

$C_{\mathrm{L}} \quad$ Lift coefficient

$C_{\mathrm{W} 1}, C_{\mathrm{W} 2}$ Wall coefficients

$d_{\mathrm{b}} \quad$ Bubble diameter (m)

$D_{\text {wall }} \quad$ Near-wall dispersion coefficient $\left(\mathrm{m}^{2} / \mathrm{s}\right)$

$D_{\text {wall }}{ }^{*} \quad$ Non-dimensional near-wall dispersion coefficient

g Gravity $\left(\mathrm{m} / \mathrm{s}^{2}\right)$

$J_{1} \quad$ Liquid superficial velocity $(\mathrm{m} / \mathrm{s})$

$J_{\mathrm{g}} \quad$ Gas superficial velocity $(\mathrm{m} / \mathrm{s})$

$M_{\mathrm{g}} \quad$ Interfacial momentum exchange term $(\mathrm{Pa} / \mathrm{m})$

$P \quad$ Pressure $(\mathrm{Pa})$

$r \quad$ Radial coordinate $(\mathrm{m})$

$R \quad$ Radius of the pipe (m)

$U_{\mathrm{R}} \quad$ Relative velocity $(\mathrm{m} / \mathrm{s})$

$V_{k} \quad$ Velocity of phase $k(\mathrm{~m} / \mathrm{s})$

$y \quad$ Distance to the wall (m)

$y^{*} \quad$ Non-dimensional distance to the wall

$z \quad$ Axial coordinate (m)

\section{GREEK LETTERS}

$\alpha_{k} \quad$ Volume fraction of phase $k$

$\delta_{\mathrm{g}} \quad$ Gas viscous layer thickness (m)

$\delta_{\mathrm{g}}{ }^{*} \quad$ Non-dimensional gas viscous layer thickness

$\mu_{k} \quad$ Viscosity of phase $k$

$\rho_{k} \quad$ Density of phase $k$

\section{SUBSCRIPTS}

$\begin{array}{ll}k & k \text {-th phase } \\ \mathrm{g} & \text { Gas phase } \\ 1 & \text { Liquid phase }\end{array}$

\section{INTRODUCTION}

The capability to understand and simulate two-phase flows is of ever-increasing importance in applications to nuclear or chemical engineering. At the same time, because of the variety of conditions and flow regimes encountered, an important Validation and Verification $(\mathrm{V} \& \mathrm{~V})$ effort is required to qualify Computational Fluid Dynamics (CFD) codes (Boyd, 2014; Podowski, 2014).
Bubbly flows involve numerous small inclusions of gas in a liquid carrier phase. Because it would be too complex to follow these structures individually, a larger-scale description is preferred. In the two-fluid formulation (Drew and Passman, 1999; Ishii and Hibiki, 2010; Morel, 2015), information on individual bubbles is eliminated through an averaging procedure. The interaction between the continuous and the dispersed phase is accounted for by closure relations. As an example, the interfacial momentum transfer is decomposed into several components: drag, added-mass, lift, dispersion and wall forces.

Many models exist for each of these forces, with different ranges of validity. These models are often derived in regions far away from the walls. However, near-wall effects can play a crucial role, especially in heat transfer phenomena, like the boiling crisis. Developing closure relations specific to the near-wall region is therefore very important.

In the literature, many models of dispersion force are dedicated to turbulent bubbly flows. They are derived in regions where velocity gradients are negligible, especially far away from the walls. In some of these models (Laviéville et al., 2015; Lopez de Bertodano, 1998), the dispersion coefficient is expressed as being proportional to the turbulent kinetic energy, and therefore vanishes at the walls.

In a recent paper (Marfaing et al., 2016), we considered the problem of a fully developed "laminar" (low Reynolds number) adiabatic bubbly flow in a vertical pipe. The dispersion force was represented by the following expression, close to Davidson's model (Davidson, 1990):

$$
\vec{M}_{\mathrm{g}}{ }^{\text {disp }}=-\frac{3}{4} \rho_{1} \frac{C_{\mathrm{D}}}{d_{\mathrm{b}}}\left|\vec{U}_{\mathrm{R}}\right| D_{\text {eff }} \nabla \vec{\alpha}_{\mathrm{g}}
$$

where $D_{\text {eff }}$ is the dispersion coefficient. Assuming $D_{\text {eff }}$ was uniform on the entire domain, and performing a balance of hydrodynamic forces on bubbles, we were able to give an analytical expression for the void fraction profile as a function of the liquid velocity and pressure profiles. The obtained analytical void fraction profile Bubble Force Balance Formula (BFBF) vanishes at the wall, as a result of the balance between dispersion and wall forces. It presents a peak near the wall for upward flows, whereas its maximum is reached at the center of the pipe for downward flows. This BFBF was then used as a verification tool for the NEPTUNE_CFD code, a Computational Multi-Fluid Dynamics (CMFD) code dedicated to the simulation of multiphase flows, primarily targeting nuclear thermalhydraulics applications, such as the Departure from Nucleate Boiling (DNB) or the two-phase Pressurized Thermal Shock (PTS). NEPTUNE_CFD has been developed within the joint NEPTUNE R\&D project (AREVA, CEA, EDF, IRSN) since 2001. 
Naturally, assuming a uniform $D_{\text {eff }}$ is a simplifying assumption. However, it is legitimate to consider the dispersion coefficient as uniform to make a comparison with experimental measurements over a localized region of space, in particular near a wall. In this region, the void fraction profile is determined by two dominant effects: dispersion and wall forces.

In this paper, we consider low Reynolds number ("laminar") bubbly flows in pipes and we investigate the void fraction profile near the walls.

In Section 1, we begin by using the NEPTUNE_CFD code to simulate a series of low Reynolds number ("laminar") adiabatic bubbly flow experiments performed by Nakoryakov et al. (1996). The calculations are seen to be in correct agreement with the experimental data. In particular, we can see that using the standard $R_{i j}-\varepsilon$ model of the code yields results for the liquid velocity fluctuations which are of the right order of magnitude.

Then, in Section 2, we focus on the near-wall region. We begin by adapting the argument in (Marfaing et al., 2016) to derive an analytical expression for the void fraction profile near the wall, where the dispersion coefficient is assumed to be uniform and non-zero. Then, the analytical profile is compared to Nakoryakov et al.'s (1996) experimental results. We find a good agreement and we can estimate the dispersion coefficient near the wall $D_{\text {wall. }}$

\section{CALCULATIONS OF "LAMINAR" AIR-WATER BUBBLY FLOW EXPERIMENTS WITH THE NEPTUNE_CFD CODE}

NEPTUNE_CFD is a Computational Multi-Fluid Dynamics (CMFD) code dedicated to the simulation of multiphase flows, which has been co-developed since 2001 in the frame of the NEPTUNE R\&D project, supported by the four major stakeholders of the French nuclear industry: $A R E V A, C E A$, $E D F$ and $I R S N$. The code is able to handle various flow regimes: stratified flows, particle- or droplet-laden flows, and bubbly flows, in order to address a variety of local phenomena occurring in a nuclear reactor in accident conditions, such as the two-phase Pressurized Thermal Shock (PTS), or the Departure from Nucleate Boiling (DNB). In order to demonstrate the applicability of CMFD codes to the prediction of these phenomena, an extensive Validation and Verification (V\&V) effort is required. In the case of DNB, this effort is focused on the assessment of the hydrodynamic models used for bubbly flows.

In this section, we use the NEPTUNE_CFD code to simulate a series of low Reynolds number ("laminar") adiabatic bubbly flow experiments. Although most experimental studies are conducted in the turbulent regime, a few "laminar" experiments are available in the literature.
Nakoryakov et al. (1996) investigated the structure of low Reynolds number bubbly flows in a vertical pipe. Their test section was a vertical cylindrical pipe with internal diameter $14 \mathrm{~mm}$ and height $6.5 \mathrm{~m}$, at the bottom of which a mixture of gas and liquid was injected.

The gas was air. The liquid used was an aqueous solution of sodium hydroxide, potassium ferri- and ferrocyanide, to which glycerine was added to increase the viscosity. In the reported experiments, the Reynolds number was comprised between 875 and 1860. For such low Reynolds numbers, the corresponding single-phase flow would be "laminar". The observed liquid velocity fluctuations ("pseudo-turbulence") are due to the presence of bubbles.

The flow was observed to be statistically steady and axisymmetric - statistically meaning that the average flow, not its fluctuations, is steady and axisymmetric.

A measuring station, located at the top of the pipe, recorded the radial profiles of void fraction, liquid velocity and velocity fluctuations. Measurements were made with an electrodiffusional method, based on the rate of mass transfer from a microelectrode to the liquid. The probe was able to get to a distance $y$ from the wall of one tenth of a bubble diameter.

Nakoryakov et al. (1996) conducted nine experimental runs called Ub101-Ub109. His experimental data are available online https://scholar.lib.vt.edu/ejournals/JFE/ data/JFE/DB96-377/. Experimental conditions are summarized in Table 1. The average bubble diameter is known at the recording station, but there is little information about the bubble size distribution. In a previous paper by the same team (Kashinsky et al., 1993), a similar test section is used and the authors report that for most of the flow regimes, the bubbles are rather uniform in size.

This experiment is simulated with the NEPTUNE_CFD code.

At present, the NEPTUNE_CFD code is devoted to the simulation of turbulent bubbly flows. No specific model for liquid velocity fluctuations in low Reynolds number flows has been implemented yet. For this reason, we make use of the standard $R_{i j}-\varepsilon$ model of the code (Mimouni et al., 2009) to account for the liquid velocity fluctuations.

In Figures 1-3, the experimental and computed profiles are compared for run Ub102.

The void fraction and liquid velocity profiles are well predicted by our simulations.

In Figure 3, the axial liquid velocity fluctuations are defined as $\sqrt{\left\langle u_{1, z}^{\prime}{ }^{2}\right\rangle}, u_{1, z}^{\prime}$ being the axial component of the liquid fluctuating velocity. We can see that using the standard $R_{i j-\varepsilon}$ model of the code yields results which are of the right order of magnitude.

For a higher gas superficial velocity (run Ub103), the same comparison is carried out in Figures 4-6. The location 
TABLE 1

Experimental runs conducted by Nakoryakov et al. (1996).

\begin{tabular}{c|c|c|c}
\hline Run number & $\begin{array}{c}\text { Liquid } \\
\text { superficial } \\
\text { velocity } \\
J_{1}(\mathrm{~m} / \mathrm{s})\end{array}$ & $\begin{array}{c}\text { Gas superficial } \\
\text { velocity } \\
J_{\mathrm{g}}(\mathrm{m} / \mathrm{s})\end{array}$ & $\begin{array}{c}\text { Bubble diameter } \\
\text { at the recording } \\
\text { station } d_{\mathrm{b}}(\mathrm{mm})\end{array}$ \\
\hline Ub101 & 0.207 & 0.0042 & 2.1 \\
\hline $\mathrm{Ub102}$ & 0.207 & 0.023 & 2.2 \\
\hline $\mathrm{Ub103}$ & 0.207 & 0.052 & 2.1 \\
\hline $\mathrm{Ub} 104$ & 0.44 & 0.0090 & 1.9 \\
\hline $\mathrm{Ub105}$ & 0.44 & 0.049 & 2 \\
\hline $\mathrm{Ub106}$ & 0.44 & 0.11 & 2.4 \\
\hline $\mathrm{Ub} 107$ & 0.44 & 0.0090 & 2 \\
\hline $\mathrm{Ub} 108$ & 0.44 & 0.049 & 3.7 \\
\hline Ub109 & 0.44 & 0.11 & 3.6 \\
\hline
\end{tabular}

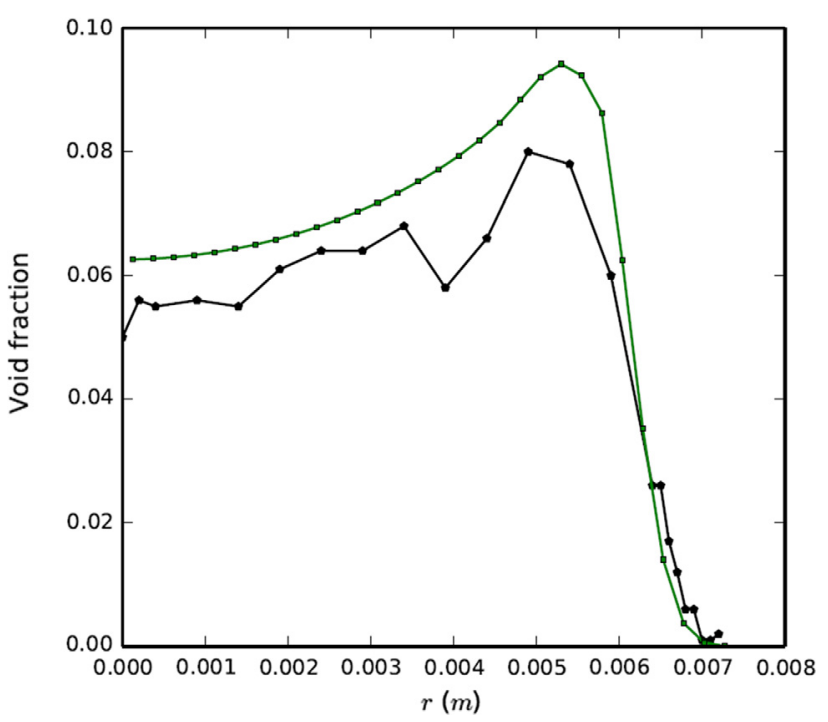

Figure 1

Computed (green) and experimental (black) void fraction profiles run Ub102.

of the void fraction peak is well captured but the calculations underestimate its magnitude: the predicted profile is too spread. The computed liquid velocity profile is in good agreement with the experiment. Again, the liquid velocity fluctuations are of the right order of magnitude, which confirms the $R_{i j}-\varepsilon$ model yields reasonable results in these low Reynolds number conditions.

Even for low Reynolds number flows, taking into account liquid velocity fluctuations is essential, because they drive

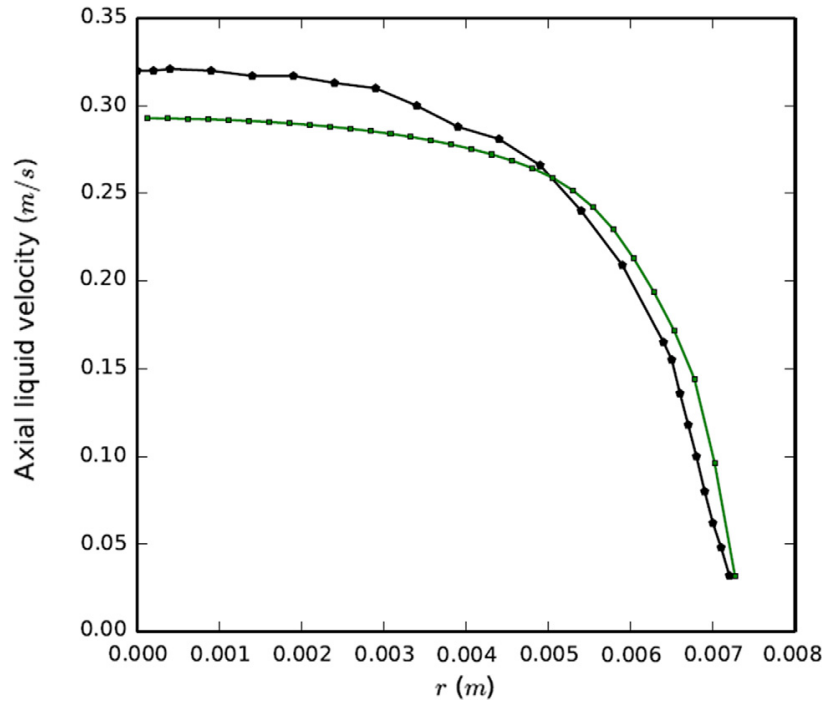

Figure 2

Computed (green) and experimental (black) liquid velocity profiles run Ub102.

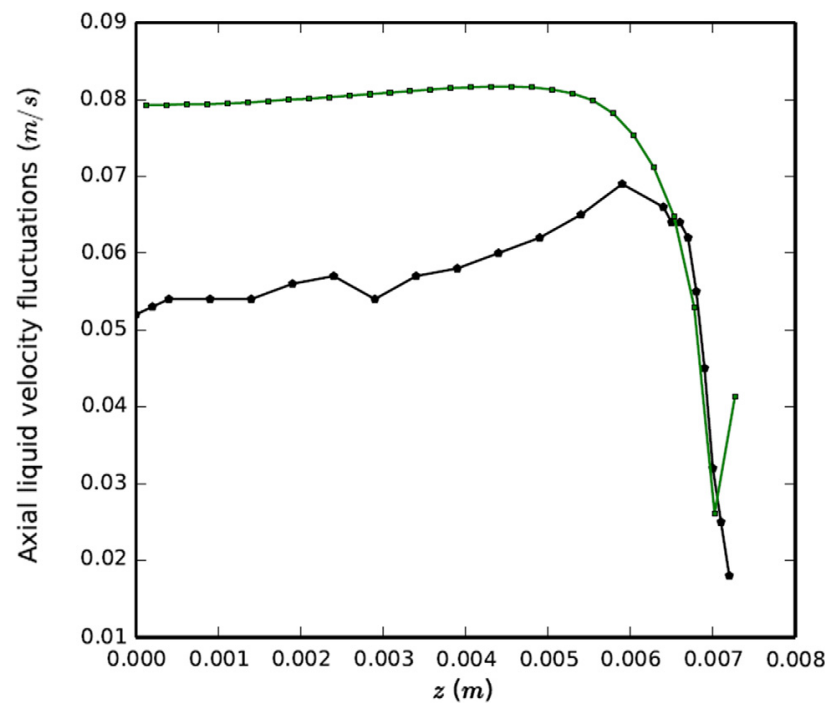

Figure 3

Computed (green) and experimental (black) axial liquid velocity fluctuations run $\mathrm{Ub} 102$.

the dispersion force. This force counter-balances the effects of lift and wall forces, and contributes to smoothing the void fraction field. For comparison, we carry out computations of run Ub102 without using a model for liquid velocity fluctuations. The obtained void fraction profile displays strong oscillations (Fig. 7). 


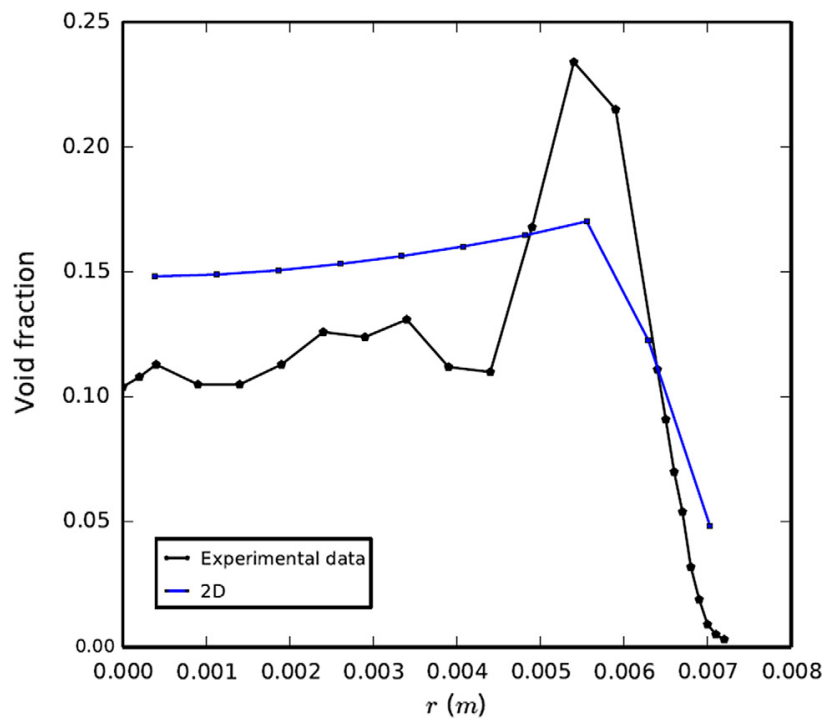

Figure 4

Computed (blue) and experimental (black) void fraction profiles run $\mathrm{Ub} 103$.

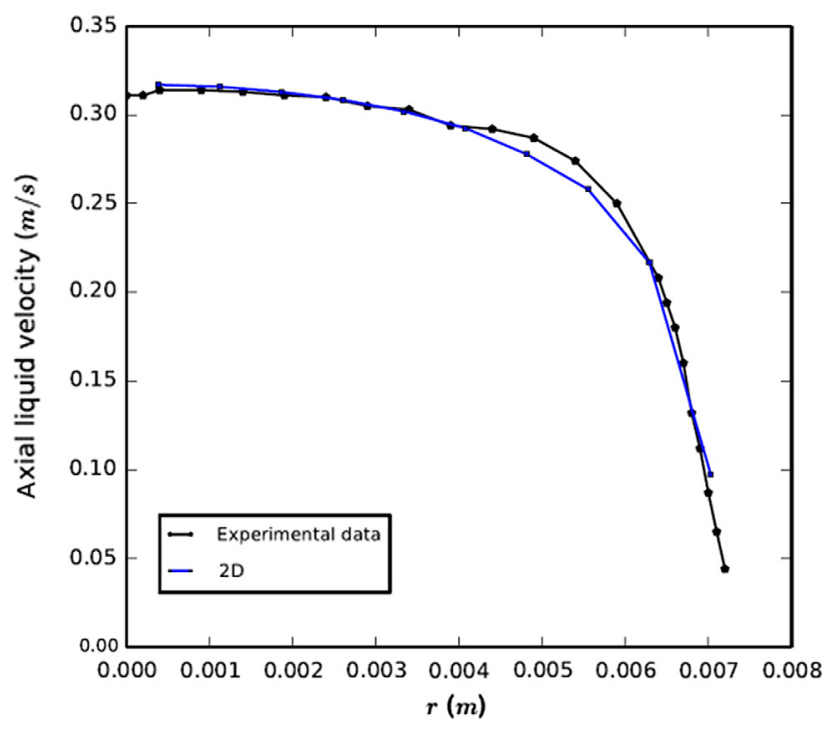

Figure 5

Computed (blue) and experimental (black) liquid velocity profiles run Ub103.

\section{ANALYTICAL AND EXPERIMENTAL NEAR-WALL VOID FRACTION PROFILES - COMPARISON - ESTIMATE OF THE WALL DISPERSION COEFFICIENT}

In this section, we focus on the near-wall void fraction profiles.

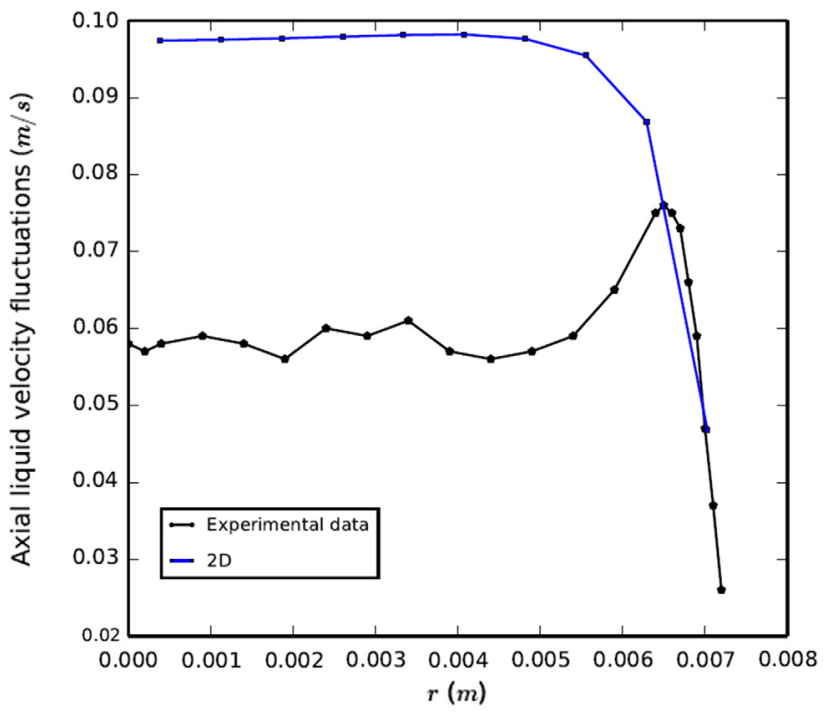

Figure 6

Computed (blue) and experimental (black) axial liquid velocity fluctuations run Ub103.

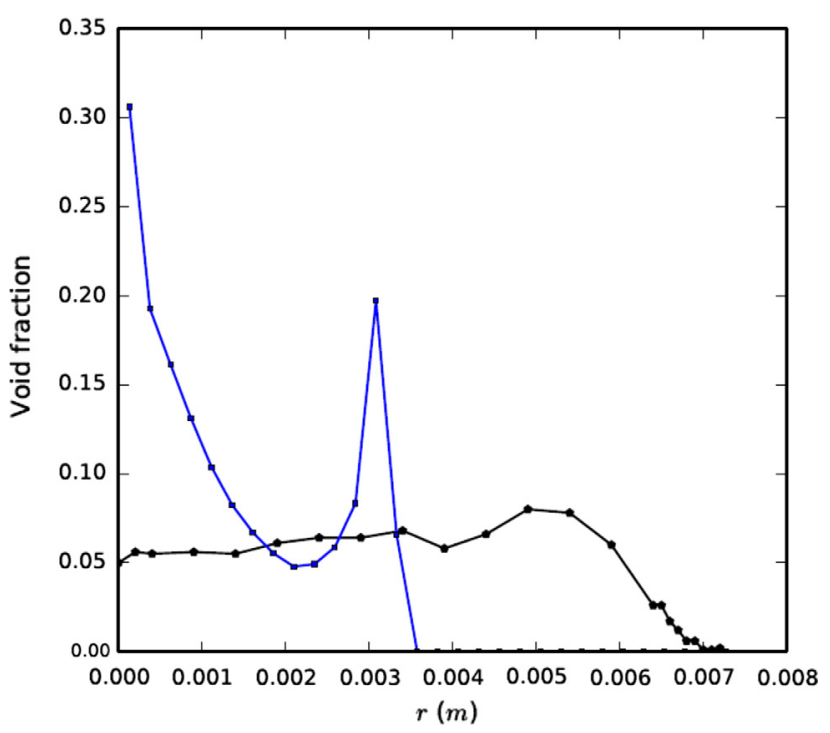

Figure 7

Computed (blue) and experimental (black) void fraction profiles run Ub102. The computations are performed without taking into account liquid velocity fluctuations.

In a recent paper (Marfaing et al., 2016), we studied analytically, with the two-fluid formalism, the problem of a fully developed "laminar" adiabatic bubbly flow in a vertical pipe. The exchange of momentum between the phases was described as the sum of drag, lift, wall and 
dispersion contributions, with uniform coefficients over the entire cross-section. Under this assumption, we were able to express analytically the void fraction distribution over the entire domain by a BFBF. This BFBF can be used as a test case for the verification of multiphase CFD codes (see BFBF case in the $\mathrm{V} \& \mathrm{~V}$ report of NEPTUNE_CFD).

The dispersion force was represented by Equation (1).

Here, in order to make experimental comparisons, we no longer assume these coefficients are uniform over the entire domain. However, it is legitimate to consider them uniform over a localized region of space, in particular near a wall.

We begin by presenting, in Section 2.1, the main ideas that lead to the derivation of an analytical expression. Some of the assumptions used in (Marfaing et al., 2016) are removed in this work. In particular, dispersion and drag coefficients are only assumed to be uniform near the wall. As a consequence, the analytical expression is restricted to the near-wall region. We show that the void fraction profile results from a balance between dispersion and wall forces.

In Section 2.2, we compare the experimental void fraction profiles obtained by Nakoryakov et al. (1996) with the analytical form derived in Section 2.1. And we use this data to estimate the dispersion coefficient at the wall.

\subsection{Analytical Near-Wall Void Fraction Profile}

Consider a low Reynolds number ("laminar") steady, axisymmetric, fully developed bubbly flow in a vertical pipe with internal radius $R$ (Fig. 8). In particular, no phase change occurs, and the variations in gas and liquid densities are neglected.

The flow being steady and fully developed, it follows from the mass balance that liquid and gas velocities $\vec{V}_{k}$ for $k=\mathrm{g}$ or 1 are purely axial and only depend on the radial coordinate $r$ :

$$
\vec{V}_{k}=V_{k}(r) \vec{e}_{z}
$$

Because the Reynolds number is low, we neglect Reynolds stresses in the gas phase, a common assumption in theoretical studies of "laminar" flows (Antal et al., 1991; Azpitarte and Buscaglia, 2003). It is also common in these studies to neglect viscous stresses in the gas phase. Strictly speaking, this may be possible only some distance away from the wall. Let $\delta_{\mathrm{g}}$ denote the thickness of the boundary layer where gas viscosity is not negligible. Denoting by $d_{\mathrm{b}}$ the bubble diameter and $y=R-r$ the distance to the wall, we assume:

$$
\delta_{\mathrm{g}} \ll d_{\mathrm{b}}
$$

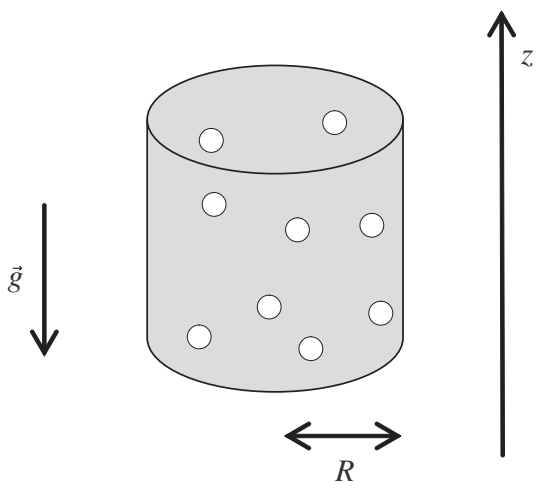

Figure 8

Bubbly flow in a vertical pipe.

and we place ourselves in the region

$$
\delta_{\mathrm{g}}<y<d_{\mathrm{b}}
$$

In this region, viscous and Reynolds stresses are neglected, and the momentum balance for the gas phase can be written:

$$
\overrightarrow{0}=-\alpha_{\mathrm{g}} \overrightarrow{\nabla P}+\alpha_{\mathrm{g}} \rho_{\mathrm{g}} \vec{g}+\vec{M}_{\mathrm{g}}
$$

where $\vec{M}_{\mathrm{g}}$ stands for the interfacial momentum exchange term. Because of Equation (2), the left-hand side of the momentum balance is zero.

The interfacial momentum transfer term $\vec{M}_{\mathrm{g}}$ can be written as the sum of four contributions (Drew and Passman, 1999; Ishii and Hibiki, 2010; Morel, 2015): drag, lift, wall, and dispersion force. Because of Equation (2), virtual mass contribution is zero. As we shall see, wall and dispersion forces are dominant.

Drag and lift forces are respectively expressed as

$$
\begin{aligned}
& \vec{M}_{\mathrm{g}}^{\mathrm{D}}=-\frac{3}{4} \alpha_{\mathrm{g}} \rho_{1} \frac{C_{\mathrm{D}}}{d_{\mathrm{b}}}\left|\vec{U}_{\mathrm{R}}\right| \vec{U}_{\mathrm{R}}, \\
& \vec{M}_{\mathrm{g}}^{\mathrm{L}}=-\alpha_{\mathrm{g}} \rho_{1} C_{\mathrm{L}} \vec{U}_{\mathrm{R}} \times\left(\overrightarrow{\operatorname{rot}} \vec{V}_{1}\right)
\end{aligned}
$$

where $C_{\mathrm{D}}$ is the drag coefficient, $d_{\mathrm{b}}$ the bubble diameter, $\vec{U}_{\mathrm{R}} \equiv \vec{V}_{\mathrm{g}}-\vec{V}_{1}$ the relative velocity between the phases, and $C_{\mathrm{L}}$ the lift coefficient. Unlike in (Marfaing et al., 2016), coefficients $C_{\mathrm{D}}$ and $C_{\mathrm{L}}$ are no longer supposed to be uniform over the entire domain. We assume the drag coefficient is uniform in the region $\delta_{\mathrm{g}}<y<d_{\mathrm{b}}$. Consequently, because gas viscosity is negligible in this region, the relative velocity is also uniform, and non-zero. 
The dispersion force, proportional to the void fraction gradient, results in the migration of bubbles from high to low void fraction regions. Unlike in (Marfaing et al., 2016), the dispersion coefficient is considered as uniform only in the region $\delta_{\mathrm{g}}<y<d_{\mathrm{b}}$, with value $D_{\text {wall }}$. In this region, the contribution of the dispersion force therefore writes:

$$
\vec{M}_{\mathrm{g}}^{\text {disp }}=-\frac{3}{4} \rho_{1} \frac{C_{\mathrm{D}}}{d_{\mathrm{b}}}\left|\vec{U}_{\mathrm{R}}\right| D_{\text {wall }} \overrightarrow{\nabla \alpha}_{\mathrm{g}}
$$

The wall force pushes the bubbles away from the wall, hence ensuring the zero void fraction condition observed experimentally. Let $\vec{n}_{\mathrm{W}}$ denote the unit vector normal to the wall pointing towards the fluid. Antal et al. (1991) express the wall force contribution as

$$
\vec{M}_{\mathrm{g}}{ }^{\mathrm{W}}=2 \alpha_{\mathrm{g}} \rho_{1} \frac{\left|\vec{U}_{\mathrm{R}}\right|^{2}}{d_{\mathrm{b}}} \operatorname{Max}\left[0, C_{\mathrm{W} 1}+C_{\mathrm{W} 2} \frac{d_{\mathrm{b}}}{2 y}\right] \vec{n}_{\mathrm{W}}
$$

Coefficients $C_{\mathrm{W} 1}$ and $C_{\mathrm{W} 2}$ are determined by Antal et al. (1991) as follows: $C_{\mathrm{W} 1}=-0.104-0.06\left|U_{\mathrm{R}}\right|$ (with the relative velocity in $\mathrm{m} / \mathrm{s}$ ) and $C_{\mathrm{W} 2}=0.147$.

The projection of Equation (5) onto the radial direction writes:

$$
\begin{aligned}
0= & -\alpha_{\mathrm{g}} \frac{\partial P}{\partial r}-\alpha_{\mathrm{g}} C_{\mathrm{L}} \rho_{1} U_{\mathrm{R}} \frac{\partial V_{1}}{\partial r} \\
& -2 \alpha_{\mathrm{g}} \rho_{1} \frac{\left|U_{\mathrm{R}}\right|^{2}}{d_{\mathrm{b}}} \operatorname{Max}\left[0, C_{\mathrm{W} 1}+C_{\mathrm{W} 2} \frac{d_{\mathrm{b}}}{2 y}\right] \\
& -\frac{3}{4} \rho_{1} \frac{C_{\mathrm{D}}}{d_{\mathrm{b}}}\left|U_{\mathrm{R}}\right| D_{\text {wall }} \frac{\partial \alpha_{\mathrm{g}}}{\partial r}
\end{aligned}
$$

Rearranging terms in Equation (9), and simplifying by the void fraction, it follows:

$$
\begin{aligned}
\frac{1}{\alpha_{\mathrm{g}}} \frac{\partial \alpha_{\mathrm{g}}}{\partial r}= & -\frac{4}{3} \frac{d_{\mathrm{b}}}{C_{\mathrm{D}} \rho_{1}\left|U_{\mathrm{R}}\right| D_{\text {wall }}} \frac{\partial P}{\partial r}-\frac{4}{3} \frac{C_{\mathrm{L}} d_{\mathrm{b}}}{C_{\mathrm{D}} D_{\text {wall }}} \frac{\partial V_{1}}{\partial r} \\
& -\frac{8}{3} \frac{\left|U_{\mathrm{R}}\right|}{C_{\mathrm{D}} D_{\text {wall }}} \operatorname{Max}\left[0, C_{\mathrm{W} 1}+C_{\mathrm{W} 2} \frac{d_{\mathrm{b}}}{2 y}\right]
\end{aligned}
$$

We recall that this expression has been derived in the region $\delta_{\mathrm{g}}<y<d_{\mathrm{b}}$, where the relative velocity is uniform and non-zero. For small distances to the wall, the dominant terms are the wall force, which goes to infinity, and the logarithmic derivative of $\alpha_{\mathrm{g}}$, because $\alpha_{\mathrm{g}}$ tends to zero. Before simplifying Equation (10), we begin by rewriting it in non-dimensional form. Let

$$
y^{*} \equiv \frac{2 y}{d_{b}}
$$

be the non-dimensional distance to the wall,

$$
V_{1}^{*} \equiv \frac{V_{1}}{\left|U_{\mathrm{R}}\right|}, P^{*} \equiv \frac{P}{\rho_{1}\left|U_{\mathrm{R}}\right|^{2}}
$$

be the non-dimensional liquid velocity and the nondimensional pressure, and

$$
D_{\text {wall }}^{*} \equiv \frac{3}{4} \frac{C_{\mathrm{D}} D_{\text {wall }}}{\left|U_{\mathrm{R}}\right| d_{\mathrm{b}}}
$$

be the non-dimensional bubble dispersion coefficient - we recall that, in the region $\delta_{\mathrm{g}}<y<d_{\mathrm{b}}$, the drag and dispersion coefficients $C_{\mathrm{D}}$ and $D_{\text {wall }}$ are constant, and the relative velocity $\left|U_{\mathrm{R}}\right|$ is constant and non-zero. Equation (10) can then be rewritten:

$$
\begin{aligned}
\frac{1}{\alpha_{\mathrm{g}}} \frac{\partial \alpha_{\mathrm{g}}}{\partial y^{*}}= & -\frac{1}{D_{\text {wall }}{ }^{*}} \frac{\partial P^{*}}{\partial y^{*}}-\frac{C_{\mathrm{L}}}{D_{\text {wall }}{ }^{*}} \frac{\partial V_{1}{ }^{*}}{\partial y^{*}} \\
& +\frac{1}{D_{\text {wall }}{ }^{*}} \operatorname{Max}\left[0, C_{\mathrm{W} 1}+\frac{C_{\mathrm{W} 2}}{y^{*}}\right]
\end{aligned}
$$

From Equation (14), the void fraction profile $\alpha_{\mathrm{g}}\left(y^{*}\right)$ depends on non-dimensional numbers which fall into three categories:

(i) non-dimensional space coordinate: $\delta_{\mathrm{g}}{ }^{*}$ defined by $\delta_{\mathrm{g}}{ }^{*} \equiv \frac{2 \delta_{\mathrm{g}}}{d_{\mathrm{b}}}$. From Equation (3), $\delta_{\mathrm{g}}{ }^{*} \ll 1$. We can neglect gas viscosity for $\delta_{g}{ }^{*}<y^{*}$,

(ii) non-dimensional dependent variables $V_{1}^{*}$ and $P^{*}$ defined by Equation (12),

(iii) non-dimensional hydrodynamic coefficients: $D_{\text {wall }}{ }^{*}$, $C_{\mathrm{L}}, C_{\mathrm{W} 1}$ and $C_{\mathrm{W} 2}$.

For small distances to the wall, the dominant terms in Equation (14) are the wall force, which goes to infinity, and the logarithmic derivative of $\alpha_{\mathrm{g}}$, because $\alpha_{\mathrm{g}}$ tends to zero. So Equation (14) can be approximated by:

$$
\frac{1}{\alpha_{\mathrm{g}}} \frac{\partial \alpha_{\mathrm{g}}}{\partial y^{*}}=\frac{C_{\mathrm{W} 2}}{D_{\text {wall }}} \frac{1}{y^{*}}
$$

This simplification reduces the number of nondimensional parameters involved in the void fraction profile $\alpha_{\mathrm{g}}\left(y^{*}\right)$ :

(i) the non-dimensional space coordinate $\delta_{\mathrm{g}}{ }^{*}$,

(ii) the ratio of hydrodynamic coefficients $C_{\mathrm{W} 2} / D_{\text {wall }}{ }^{*}$.

Integration of (15) yields the near-wall void fraction profile:

$$
\alpha_{\mathrm{g}}=B \times\left(y^{*}\right)^{C_{\mathrm{W}_{2}} / D_{\text {wall }}}{ }^{*}
$$


where $B$ is a non-dimensional integration constant, depending on the gas flow rate. This expression is of course consistent with the BFBF derived in (Marfaing et al., 2016). However, some of the assumptions used in (Marfaing et al., 2016) are removed in the derivation of Equation (16).

In Section 2.2, we compare Equation (16) with Nakoryakov et al.'s (1996) measurements. We will also be able to estimate the dispersion coefficient.

\subsection{Experimental Comparison - Estimate of the Wall Dispersion Coefficient}

For each run carried out by Nakoryakov et al. (1996), we examine the void fraction measurements in the region $y<d_{\mathrm{b}}, y$ standing for the distance to the wall, and $d_{\mathrm{b}}$ for the bubble diameter. In order to test Equation (16), we plot the graph of the logarithm of the void fraction $\ln \alpha_{\mathrm{g}}$ as a function of $\ln y^{*}$. We calculate the least-squares line and the correlation coefficient $R^{2}$. The example of run Ub103 is displayed in Figure 9. It must be noted that for this run, the measured void fraction vanishes nowhere.

Figure 9 reveals that the nine experimental points of run Ub103 are almost aligned. The correlation coefficient $R^{2}$ is $97 \%$ and the slope of the least-squares line is 2.1 . So the void fraction profile in the near-wall region is satisfactorily represented by Equation (16), with $C_{\mathrm{W} 2} / D_{\text {wall }}{ }^{*}=2.1$. Using the value $C_{\mathrm{W} 2}=0.15$ recommended by Antal et al. (1991), we can estimate the non-dimensional dispersion coefficient as $D_{\text {wall }}{ }^{*}=0.070$.

The same procedure is applied for the other runs.

No zero values of the void fraction were reported by the experimenters for runs Ub102, Ub103, Ub106, so it is possible to calculate the logarithm of the void fraction $\ln \alpha_{\mathrm{g}}$.

Experimental profiles Ub105, Ub108 and Ub109 present isolated points ( 2 out of 9 ) where the reported void fraction is zero. This is illustrated in Table 2, which shows the experimental values of $\alpha_{\mathrm{g}}$ as a function of $y^{*}$ in the case of run Ub105.

In view of the recorded values, we can assume that for $y^{*}=0.2$ or 0.3 , the void fraction in the experiment was not strictly zero but too low to be detected by the probe.

In runs Ub101, Ub104 and Ub107, the gas flow rate at the bottom of the pipe is very low (see Tab. 1). For this reason, the recorded values of void fraction are one order of magnitude lower than in the other cases, and half the points were 0 . This is illustrated in Table 3, which shows the experimental values of $\alpha_{\mathrm{g}}$ as a function of $y^{*}$ in the case of run Ub107.

Again, we can reasonably assume that void fractions below 0.001 were not detected by the probe.

Consequently, our analysis for runs Ub101, Ub104, Ub105, Ub107, Ub108 and Ub109 is conducted without taking into account these zero values.

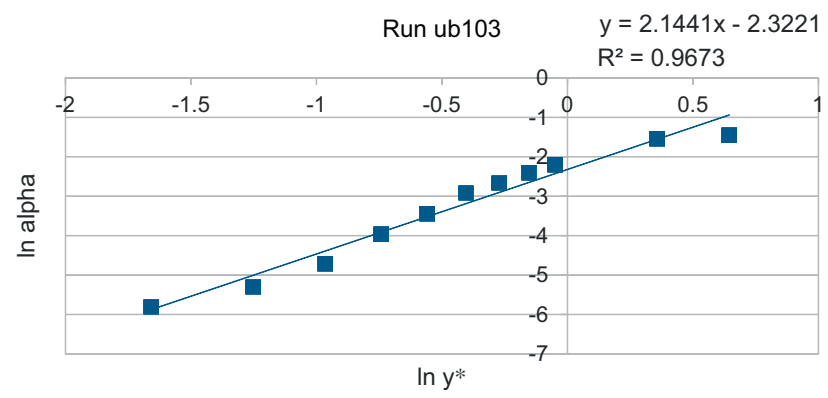

Figure 9

Graph of $\ln \alpha_{\mathrm{g}}$ as a function of $\ln y^{*}$ for run Ub103. The squares are the experimental points, and the solid line is the calculated least-squares line.

The example of run Ub105 and Ub107 is displayed in Figures 10 and 11.

In Table 4, we sum up the results of this procedure for all runs.

High correlation coefficients $R^{2}$ (above $87 \%$ ) are found for all runs. So the void fraction profile in the near-wall region is satisfactorily represented by our analytical model (Eq. 16). The non-dimensional dispersion coefficient $D_{\text {wall }}{ }^{*}$ keeps the same order of magnitude over all runs, and lies in the range $0.053-0.082$.

\subsection{Discussion}

As explained in Introduction, most existing models of dispersion force are dedicated to turbulent bubbly flows. They are derived in regions where velocity gradients are negligible, especially far away from the walls. In some of these models, the dispersion coefficient is expressed as being proportional to the turbulent kinetic energy of the liquid phase, and vanishes at the walls.

In our work, the focus is on low Reynolds number flows, and we show that assuming a non-zero dispersion coefficient near the wall leads to good predictions when compared to the experiments.

The present experimental comparison is based on a series of nine experimental runs in "laminar" conditions. Additional measurements over a wider range of conditions would be required to examine the dependence of $D_{\text {wall }}{ }^{*}$ on the flow parameters. Direct Numerical Simulations (DNS) may also help, taking advantage of the low Reynolds numbers involved.

We can conjecture an important parameter the dispersion coefficient depends on is the wall shear - or to be precise the non-dimensional shear $\left.\frac{\partial V^{*}}{\partial y^{*}}\right|_{y^{*}=0}$. Understanding this 
TABLE 2

Near-wall void fraction measurements for run Ub105.

\begin{tabular}{c|c|c|c|c|c|c|c|c|c}
\hline$y^{*}$ & 0.2 & 0.3 & 0.4 & 0.5 & 0.6 & 0.7 & 0.8 & 0.9 & 1 \\
\hline$\alpha_{g}$ & 0 & 0 & 0.003 & 0.008 & 0.01 & 0.021 & 0.035 & 0.052 & 0.077 \\
\hline
\end{tabular}

TABLE 3

Near-wall void fraction measurements for run Ub107.

\begin{tabular}{c|c|c|c|c|c|c|c|c|c|c}
\hline$y^{*}$ & 0.2 & 0.3 & 0.4 & 0.5 & 0.6 & 0.7 & 0.8 & 0.9 & 1 & 1.5 \\
\hline$\alpha_{g}$ & 0 & 0 & 0 & 0 & 0 & 0.001 & 0.001 & 0.001 & 0.002 & 0.003 \\
\hline
\end{tabular}

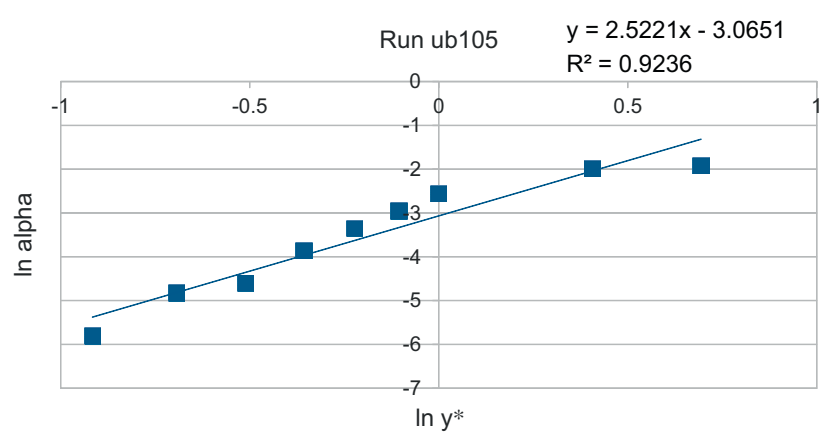

Figure 10

Graph of $\ln \alpha_{\mathrm{g}}$ as a function of $\ln y^{*}$ for run Ub105. The squares are the experimental points, and the solid line is the calculated least-squares line.

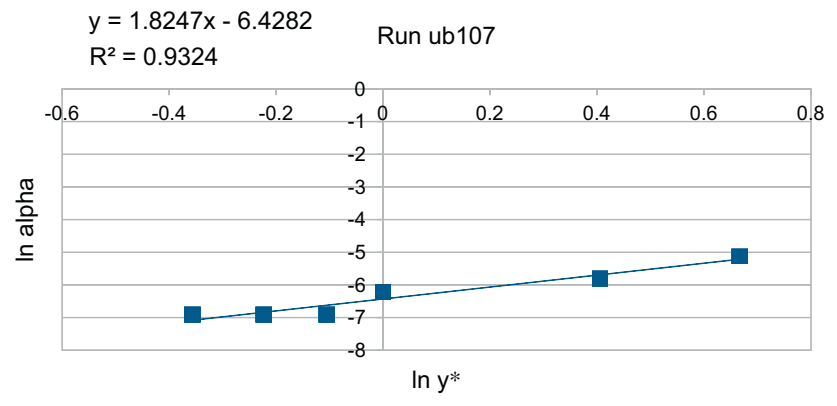

Figure 11

Graph of $\ln \alpha_{\mathrm{g}}$ as a function of $\ln y^{*}$ for run Ub107. The squares are the experimental points, and the solid line is the calculated least-squares line.

TABLE 4

Evaluation of the non-dimensional dispersion coefficient $D_{\text {eff }}{ }^{*}$. Constant $C_{\mathrm{W} 2}$ is set to 0.15 .

\begin{tabular}{c|c|c|c|c|c|c|c|c|c}
\hline & Ub101 & Ub102 & Ub103 & Ub104 & Ub105 & Ub106 & Ub107 & Ub108 & Ub109 \\
\hline Correlation coefficient $R^{2}$ & 0.99 & 0.87 & 0.97 & 0.89 & 0.92 & 0.97 & 0.93 & 0.95 & 0.97 \\
\hline Least-squares slope $C_{\mathrm{W} 2} / D_{\text {wall }}{ }^{*}$ & 2.8 & 2.1 & 2.1 & 2 & 2.5 & 2.8 & 1.8 & 1.8 & 2.5 \\
\hline Non-dimensional dispersion coefficient $D_{\text {wall }}{ }^{*}$ & 0.053 & 0.070 & 0.070 & 0.074 & 0.059 & 0.053 & 0.082 & 0.082 & 0.059 \\
\hline
\end{tabular}

relationship is probably easier in low Reynolds number flows. The knowledge gained could shed some light on more general situations, including the high Reynolds number case - existing high Reynolds number dispersion models being developed for the limit case of no shear.

"Laminar" bubbly flows have been given less attention than turbulent flows in the literature, and for the above reasons, it is important to conduct more experiments in this regime.

Having a wider experimental database would also help assess the possible effect of the bubble size distribution. As mentioned in Section 1, Nakoryakov et al. (1996) measure the average bubble diameter at the recording station. There is little information about the distribution of bubble diameters, but in a previous paper by the same team (Kashinsky et al., 1993), a similar test section is used and the authors report that for most of the flow regimes, the bubbles are rather uniform in size.

\section{CONCLUSION}

In this paper, we consider low Reynolds number ("laminar") bubbly flows in pipes and we investigate the void fraction profile near the walls. 
We begin by using the NEPTUNE_CFD code to simulate a series of low Reynolds number ("laminar") adiabatic bubbly flow experiments performed by Nakoryakov et al. (1996). The calculations are seen to be in correct agreement with the experimental data. In particular, we can see that using the standard $R_{i j}-\varepsilon$ model of the code yields results for the liquid velocity fluctuations that are of the right order of magnitude.

In the second part, we focus on the near-wall region. We begin by adapting the argument in Marfaing et al. (2016) to derive an analytical expression for the void fraction profile in the local region $\delta_{\mathrm{g}}<y<d_{\mathrm{b}}$, where the dispersion coefficient is assumed to be uniform and nonzero, and where the gas viscosity and Reynolds stresses are neglected. In this region, the void fraction profile is found to result from a balance between wall and dispersion forces.

Then, the analytical profile is compared to Nakoryakov et al.'s (1996) experimental results. High correlation coefficients $R^{2}$ (above $87 \%$ ) are found for all runs, thus validating the analytical derivation. At the same time, the calculated least-squares lines enable us to evaluate the non-dimensional coefficient $D_{\text {wall }}{ }^{*}$ to be used in our dispersion force model. $D_{\text {wall }}{ }^{*}$ is consistently found to be about 0.07 for the considered runs.

It would be interesting in future work to study the dependence of $D_{\text {wall }}{ }^{*}$ on the flow conditions. "Laminar" bubbly flows have been given less attention than turbulent flows in the literature. So it would be important to carry out more experiments.

It is also important to mention that, in the literature, most models of dispersion force are dedicated to turbulent bubbly flows. They are derived in regions where velocity gradients are negligible, especially far away from the walls. In some of these models, the dispersion coefficient is expressed as being proportional to the turbulent kinetic energy of the liquid phase, and vanishes at the walls. Here, on the contrary, the use of a non-zero dispersion coefficient is shown to yield good predictions. Although our comparisons have been carried out for "laminar" flows, we believe that using a dispersion coefficient which does not vanish at the wall is also relevant in turbulent flows.

\section{ACKNOWLEDGMENTS}

This work was performed in the frame of the NEPTUNE Project, financially supported by CEA (Commissariat à l'Énergie Atomique), EDF (Électricité de France), IRSN
(Institut de Radioprotection et de Sûreté Nucléaire) and AREVA-NP.

\section{REFERENCES}

Antal S.P., Lahey R.T., Flaherty J.E. (1991) Analysis of phase distribution in fully developed laminar bubbly two-phase flow, Int. J. Multiphase Flow 17, 5, 635-652.

Azpitarte O.E., Buscaglia G.C. (2003) Analytical and numerical evaluation of two-fluid model solutions for laminar fully developed bubbly two-phase flows, Chem. Eng. Sci. 58, 3765-3776.

Boyd C. (2014) Perspectives on CFD analysis in nuclear reactor regulation, Keynote Lecture, CFD4NRS-5 Conference, 9-11 September, Zurich, Switzerland.

Davidson M.R. (1990) Numerical calculations of two-phase flow in a liquid bath with bottom gas injection: the central plume, Appl. Math. Modelling 14, 67-76.

Drew D.A., Passman S.L. (1999) Theory of multicomponent fluids, Springer, New York, ISBN 0-387-98380-5.

Ishii M., Hibiki T. (2010) Thermo-fluid dynamics of two-phase flows, Springer, New York.

Kashinsky O.N., Timkin L.S., Cartellier A. (1993) Experimental study of "laminar" bubbly flows in a vertical pipe, Exp. Fluids 14, 308-314.

Laviéville J., Mérigoux N., Guingo M., Baudry C., Mimouni S. (2015) A generalized turbulent dispersion model for bubbly flow numerical simulation in NEPTUNE_CFD, Proc. NURETH-2015 Conference, August 30-September 4, 2015, Chicago.

Lopez de Bertodano M. (1998) Two-fluid model for two-phase turbulent jet, Nucl. Eng. Des. 179, 11, 65-74.

Marfaing O., Guingo M., Laviéville J., Bois G., Méchitoua N., Mérigoux N., Mimouni S. (2016) An analytical relation for the void fraction distribution in a fully developed bubbly flow in a vertical pipe, Chem. Eng. Sci. 152, 579-585.

Mimouni S., Archambeau F., Boucker M., Laviéville J., Morel C. (2009) A second-order turbulence model based on a Reynolds stress approach for two-phase flow - part I: adiabatic cases, Sci. Technol. Nucl. Install. 2009, 792395.

Morel C. (2015) Mathematical modeling of disperse two-phase flows, Springer, New York.

Nakoryakov V.E., Kashinsky O.N., Randin V.V., Timkin L.S. (1996) Gas-liquid bubbly flows in vertical pipes, J. Fluids Eng. 118, 377-382, Experimental data are available online https:// scholar.lib.vt.edu/ejournals/JFE/data/JFE/DB96-377/.

Podowski M.Z. (2014) Model verification and validation issues for multiphase flow and heat transfer simulation in reactor systems, Keynote Lecture, CFD4NRS-5 Conference, 9-11 September, Zurich, Switzerland.

\footnotetext{
Manuscript submitted in September 2016

Manuscript accepted in December 2016

Published online in February 2017
}

Cite this article as: O. Marfaing, M. Guingo, J.-M. Laviéville and S. Mimouni (2017). Analytical Void Fraction Profile Near the Walls in Low Reynolds Number Bubbly Flows in Pipes: Experimental Comparison and Estimate of the Dispersion Coefficient, Oil Gas Sci. Technol 72, 4. 